\title{
LUNAR OCCULTATIONS OF THE SIO MASERS IN RLEO
}

\author{
J. CERNICHARO
}

Centro Astronómico de Yebes. Apartado 148. 19080 Guadalajara. Spain

\author{
W. BRUNSWIG, G. PAUBERT, AND S. LIECHTI \\ IRAM. Av. Divina Pastora N7, NC. 18012 Granada. Spain
}

\section{INTRODUCTION}

VLBI observations show that the $\mathrm{SiO}$ maser emitting regions in oxygen-rich stars are very clumpy and that these clumps extend over a few stellar radii (McIntosh et al. 1987; Colomer et al. 1992). These observations indicate that the ideal instrument for the study of the $\mathrm{SiO}$ masers is an interferometer with baselines covering between a few and several hundreds/thousands km. Such an instrument is so far unavailable.

A classical way to get high angular resolution and a full beam synthesis with a single telescope of moderate size is through lunar occultations. This observing technique provides the angular resolution of a single linear antenna several kilometers long. However, at millimeter wavelengths the Fresnel fringes produced by the Moon limb as the source under study is occulted have never been observed. We present here the observation with the $30-\mathrm{m}$ IRAM radio telescope of the $\mathrm{v}=1 \mathrm{~J}=2-1$ line of $\mathrm{SiO}$ during an occultation (and reappearance) of $\mathrm{R}$ Leo by the Moon.

\section{OBSERVATIONS AND RESULTS}

Two maser lines, the $J=3-2$ and the $J=2-1$ of the first vibrational state of SiO were observed simultaneously with the 30 -m IRAM radio telescope during an occultation of R Leo by the Moon on May 1990. The spectrometers were 2x128 filters of $100 \mathrm{kHz}$ and $2 \times 256$ of $1 \mathrm{MHz}$. The receiver SSB temperature was 130 and $110 \mathrm{~K}$ for the $3 \mathrm{~mm}$ and $2 \mathrm{~mm}$ receivers and the sky opacity at these frequencies was 0.1 . The total main beam system temperature, including the Moon contribution, was $600 \mathrm{~K}$. The adopted integration time was 50 milliseconds during the immersion and emersion of the source. The OFFs for each spectrum of the occultation and reappearance were obtained with an integration time of 2 minutes before immersion and emersion of the source. The measured r.m.s. in each spectrum was $8.5 \mathrm{~K}$ and $2.7 \mathrm{~K}$ for the $100 \mathrm{kHz}$ and $1 \mathrm{MHz}$ filters respectively. Fresnel fringes were observed for all the velocities of the maser emission.

The adopted restoration method was that proposed by Scheuer (1962). Some restored brightness distributions are shown in Figure 1. A position-velocity diagram is shown in Figure 2. Positions are relative to the velocity of $-0.1 \mathrm{kms}^{-1}$ and all restored brigthness spatial distributions have been normalized to unity. This Figure shows that the $\mathrm{SiO} J=2-1 \mathrm{v}=1$ maser emission arises from a region 
50 mas wide and with a clear separation in space of the emission at extreme velocities. The difference in velocity between the emission at positions +25 mas and -25 mas is of $4 \mathrm{kms}^{-1}$. The picture shown in this Figure suggests a rotation of the gas where the maser emission is produced. Assuming a distance of $150 \mathrm{pc}$ for R Leo (see McIntosh 1987) the observed angular size corresponds to a linear dimension of $1.110^{14} \mathrm{~cm}$. The stellar radius is given by McIntosh to be $2.310^{13}$ $\mathrm{cm}$ which yields a total size for the maser emitting region of 5 stellar radii. A detailed description and analysis of the data will be published elsewhere.

Acknowledgments: This work has been partially supported by CICYT under project number PB90-408.

\section{REFERENCES}

Colomer et al. 1982, Astron. Astrophys. Letters, 254, L17.

McIntosh G.C. 1987, Ph.D., University of Massachusetts.

Scheuer P.A.G. 1962, Australian J. Phys., 15, 333.
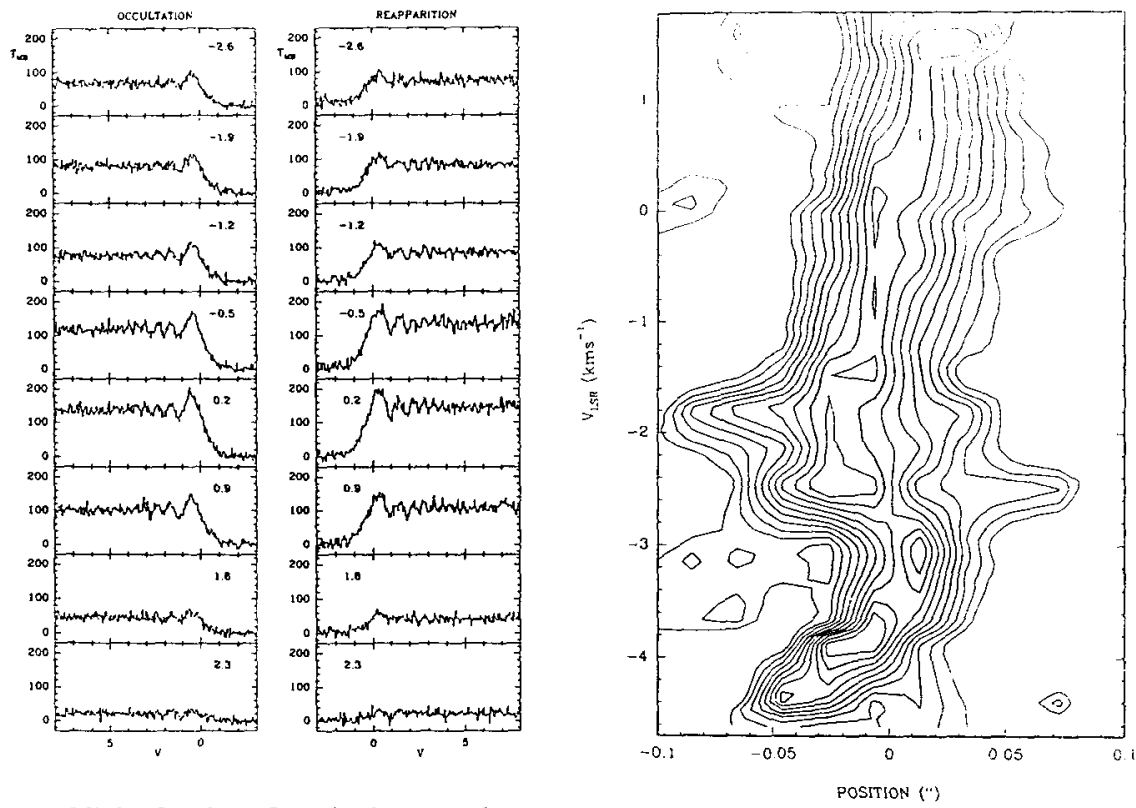

FIGURE 1: Occultation and reappearance of the $\mathrm{SiO}$ masers at several velocities. $\mathrm{X}$-axis represents the distance of the source to the occultation point in Fresnel units $\left(1 \mathrm{v} \approx 0.4^{\prime \prime}\right)$.

FIGURE 2: A velocity-position diagramm showing a systematic velocity gradient between -2.5 and $1.5 \mathrm{kms}^{-1}$. The angular separation between extreme velocities is of 50 mas. $X$-axis represents the position along the occultation path. 